\title{
Motor Manifestations
}

National Cancer Institute

\section{Source}

National Cancer Institute. Motor Manifestations. NCI Thesaurus. Code C5039.

The presence of an alteration in the ability to move one's body or any body parts in accordance with one's intent. 